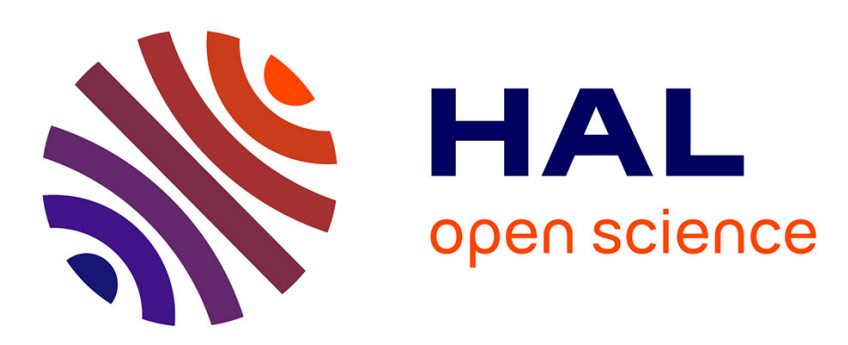

\title{
A simple predistortion technique for suppression of nonlinear effects in periodic signals generated by nonlinear transducers
}

\author{
Antonin Novak, Laurent Simon, Pierrick Lotton
}

\section{To cite this version:}

Antonin Novak, Laurent Simon, Pierrick Lotton. A simple predistortion technique for suppression of nonlinear effects in periodic signals generated by nonlinear transducers. Journal of Sound and Vibration, 2018, 420, pp.104-113. 10.1016/j.jsv.2018.01.038 . hal-02504399

\section{HAL Id: hal-02504399 \\ https://hal.science/hal-02504399}

Submitted on 10 Mar 2020

HAL is a multi-disciplinary open access archive for the deposit and dissemination of scientific research documents, whether they are published or not. The documents may come from teaching and research institutions in France or abroad, or from public or private research centers.
L'archive ouverte pluridisciplinaire HAL, est destinée au dépôt et à la diffusion de documents scientifiques de niveau recherche, publiés ou non, émanant des établissements d'enseignement et de recherche français ou étrangers, des laboratoires publics ou privés. 


\title{
A simple predistortion technique for suppression of nonlinear effects in periodic signals generated by nonlinear transducers.
}

\author{
Antonin Novak*, Laurent Simon, Pierrick Lotton \\ Laboratoire d'Acoustique de I'Université du Mans, LAUM - UMR 6613 CNRS, Le Mans Université, Avenue Olivier \\ Messiaen, 72085 LE MANS CEDEX 9, France \\ *antonin.novak@univ-lemans.fr, *https://ant-novak.com
}

\begin{abstract}
Mechanical transducers, such as shakers, loudspeakers and compression drivers that are used as excitation devices to excite acoustical or mechanical nonlinear systems under test are imperfect. Due to their nonlinear behaviour, unwanted contributions appear at their output besides the wanted part of the signal. Since these devices are used to study nonlinear systems, it should be required to measure properly the systems under test by overcoming the influence of the nonlinear excitation device. In this paper, a simple method that corrects distorted output signal of the excitation device by means of predistortion of its input signal is presented. A periodic signal is applied to the input of the excitation device and, from analysing the output signal of the device, the input signal is modified in such a way that the undesirable spectral components in the output of the excitation device are cancelled out after few iterations of real-time processing. The experimental results provided on an electrodynamic shaker show that the spectral purity of the generated acceleration output approaches $100 \mathrm{~dB}$ after few iterations (one second). This output signal, applied to the system under test, is thus cleaned from the undesirable components produced by the excitation device; this is an important condition to ensure a correct measurement of the nonlinear system under test.
\end{abstract}

The archived file is not the final published version of the article A. Novak, L. Simon \& P. Lotton (2018), "A simple predistortion technique for suppression of nonlinear effects in periodic signals generated by nonlinear transducers", Journal of Sound and Vibration. Vol. 420, pp. 104-113.

The definitive publisher-authenticated version is available online at $\mathrm{https}: / / \mathrm{doi} . \mathrm{org} / 10.1016 / \mathrm{j} \cdot \mathrm{jsv} .2018 .01 .038$, Readers must contact the publisher for reprint or permission to use the material in any form. 


\section{Introduction}

Consider a nonlinear physical system under test whose input signal $x(t)$ (displacement, mechanical force, acoustical pressure,...$)$ is generated by an excitation device (shaker, compression driver, loudspeaker, ...). If a high excitation level is applied, which is usually the case in order to emphasize the nonlinear phenomena of the system under test, the level of nonlinearities generated by the excitation device can not be considered as negligible [1,2]. The excitation device used for the measurement is usually driven by an electrical signal $u(t)$ created either by a signal generator or by a Data Acquisition (DAQ) device that enables an easy generation of signals. A general block diagram of such measurement setup using a DAQ is depicted in Fig. 1. For a linear excitation device, if the input signal $u(t)$ is a sine signal, the output $x(t)$ will also be a sine signal at exactly the same frequency. However, if the excitation device is nonlinear, added frequency components with frequencies equal to multiple integers of the fundamental input frequency can appear at the output signal $x(t)$ (the effect being called harmonic distortion) as schematically depicted in Fig. 1. If the input signal $u(t)$ consists of several harmonic components, harmonic distortion of each component and inter-modulation distortion between each pair of components can appear in the output signal $x(t)$. Consequently, the output signal $y(t)$ of the system under test will be distorted by both nonlinear systems: the excitation device and the system under test.

Since many experimental and theoretical studies are focused on nonlinear acoustics and nonlinear vibration systems $[3,4]$, and for which the experiments are conducted under high-amplitude acoustic wave [5, 6] or largeamplitude vibrations $[7,8]$, a need to ensure a non-distorted signal at the output of the excitation device is very important. To overcome the difficulties caused by a nonlinear excitation device, a technique that correct the excitation signal (based on adaptive $[9,10,11,12,13,14,15,16,17,18]$, control $[19,20,21, ?, 22,23]$, or harmonic injection $[24,25]$ methods), or a technique that takes into account the nonlinearities of the excitation device [26] must be used.

In this paper, we show that using a very simple approach inspired by [27], spectrally pure signals can be generated at the output of a nonlinear mechanical excitation device using a real-time signal processing implemented on a

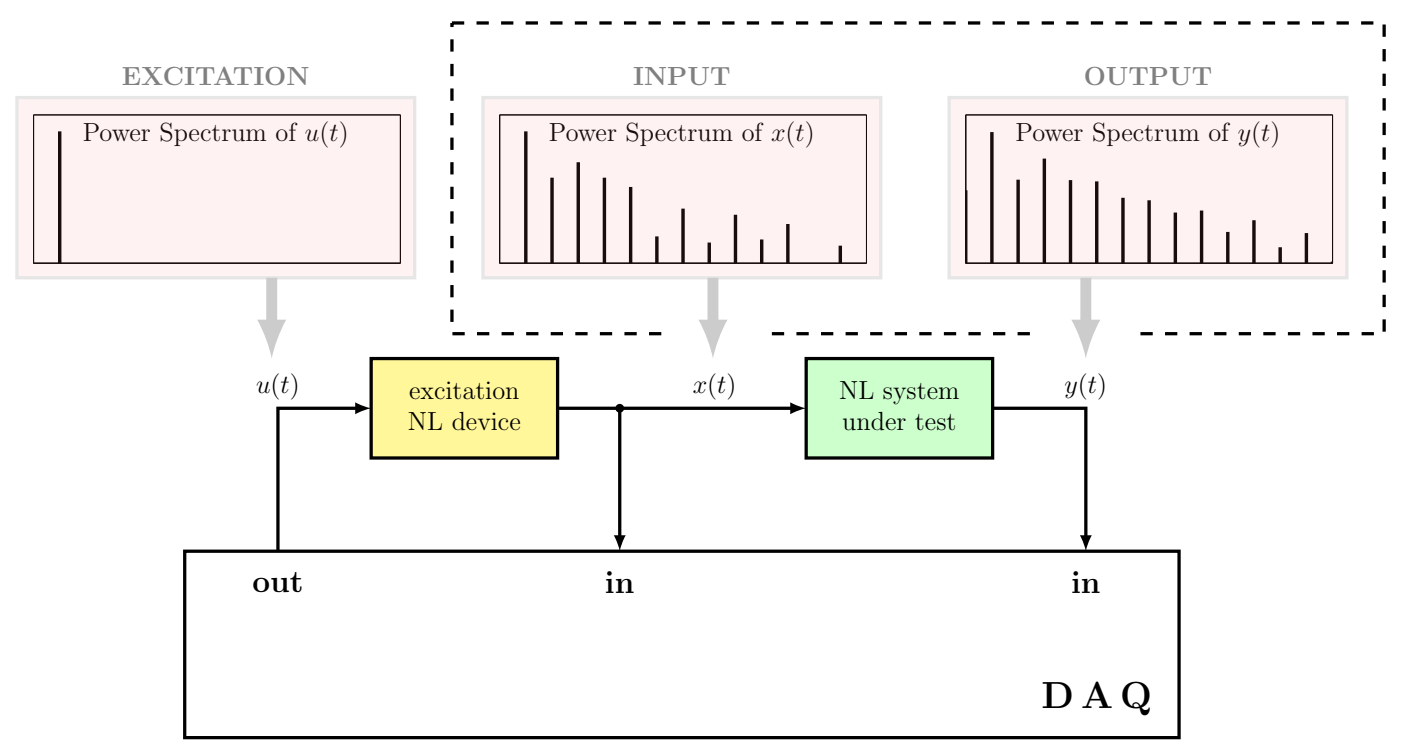

Figure 1. Measurement setup of a nonlinear experimental bench. A high-level input signal $u(t)$ is applied to the excitation device (shaker, loudspeaker, ...). Due to its nonlinear behavior higher harmonics and/or inter-modulation products are added to the signal $x(t)$ generated by the device. The output $y(t)$ of the NL system under test thus contains nonlinear components of both devices. 
personal computer and a data acquisition device (DAQ). This method is based on hypothesis that the excitation device has a dominant linear path. Such devices behave linearly for low amplitudes and become nonlinear for higher amplitudes - as usually observed in case of loudspeakers, shakers, or compression drivers. A periodic signal (sinusoidal or multi-harmonic excitation) is applied to the excitation device, the signal generated by the device is acquired and analysed and the input signal of the excitation device is adaptively modified in such a way that the undesirable spectral peaks in the output of the excitation device are completely cancelled out after few iterations of real-time processing. The signal applied to the system under test is thus cleaned from the undesirable components produced by the excitation device, allowing an accurate measurement of the nonlinear system under test with no influence of the nonlinearities of the excitation device.

The predistortion algorithm and a its theoretical description are described in Sec. 2. The method is next experimentally verified in Sec. 3 for an electrodynamic shaker which produces a high-amplitude acceleration, measured by an accelerometer, with a spectral purity approaching $100 \mathrm{~dB}$. A discussion on the efficiency, applicability, advantages and drawbacks of the method is finally proposed in Sec. 4.

\section{Predistortion of the Excitation Signal $u(t)$}

\subsection{Theoretical description of the predistortion}

The basic idea of the predistortion consists in modifying the amplitude $U_{m}$ and the phase $\phi_{m}$ of the harmonic components (including the DC component $U_{0}$ ) of the signal $u(t)$ generated by the DAQ (Fig. 2)

$$
u(t)=U_{0}+\sum_{m=1}^{M} U_{m} \cos \left(m 2 \pi f_{0} t+\phi_{m}\right),
$$

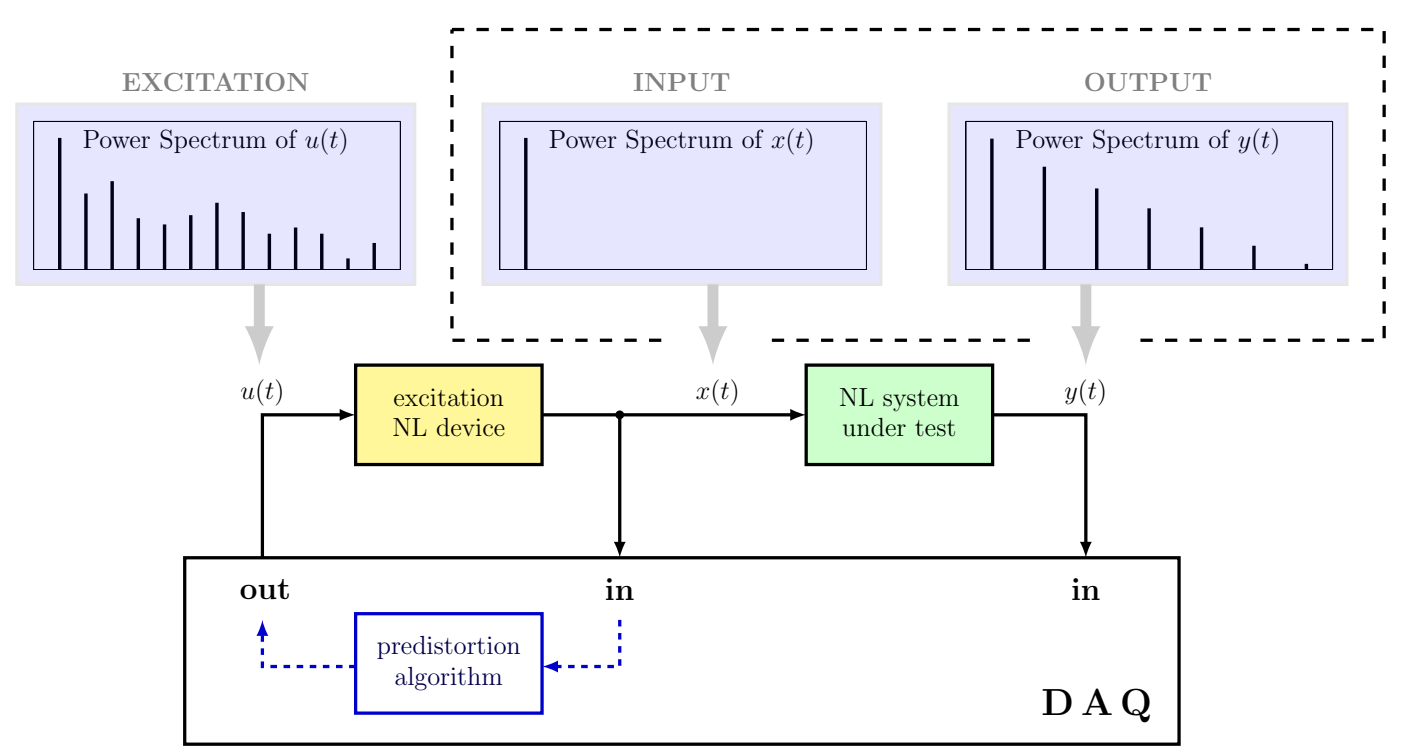

Figure 2. Measurement setup of a nonlinear experimental bench using the predistortion technique. The input signal $u(t)$, applied to the excitation device (shaker, loudspeaker, ...), is predistorted in such a way that the output signal $x(t)$ generated by the device contains only the desired part of the signal. Contrary to the setup from Fig. 1, the output $y(t)$ thus contains nonlinear components generated only by the NL system under test. 
$f_{0}$ being the fundamental frequency and $M$ being the number of harmonics, in such a way that the measured amplitudes $X_{m}$ and phases $\psi_{m}$ of the harmonic components of the signal $x(t)$

$$
x(t)=X_{0}+\sum_{m=1}^{M} X_{m} \cos \left(m 2 \pi f_{0} t+\psi_{m}\right),
$$

are equal to the desired amplitudes $X_{m}^{\oplus}$ and phases $\psi_{m}^{\oplus}$ of the harmonic components of the so-called target signal $x^{\oplus}(t)$ (the symbol $\oplus$ being used as a target marker)

$$
x^{\oplus}(t)=X_{0}^{\oplus}+\sum_{m=1}^{M} X_{m}^{\oplus} \cos \left(m 2 \pi f_{0} t+\psi_{m}^{\oplus}\right) .
$$

In the specific case of a pure sine wave generation $(\oplus \sin )$, the goal is to add higher harmonics to the input signal $u(t)$ so that the harmonics contained in the signal $x(t)$ at the output of the excitation device are suppressed, the only component present in this signal $x(t)$ being then the fundamental harmonic with desired target amplitude $A_{1}^{\oplus}$ and phase $\varphi_{1}^{\oplus}$, as

$$
x^{\oplus \sin }(t)=A_{1}^{\oplus} \cos \left(2 \pi f_{0} t+\varphi_{0}^{\oplus}\right)+e(t) .
$$

In practice, an error signal $e(t)$ modeling both the noise of the measurement setup and a possible residual of harmonics is present. The block diagram of this procedure, including the predistortion block, is depicted in Fig. 2, with corresponding power spectra in the case of a pure sine wave generation.

\subsection{Detailed predistortion algorithm}

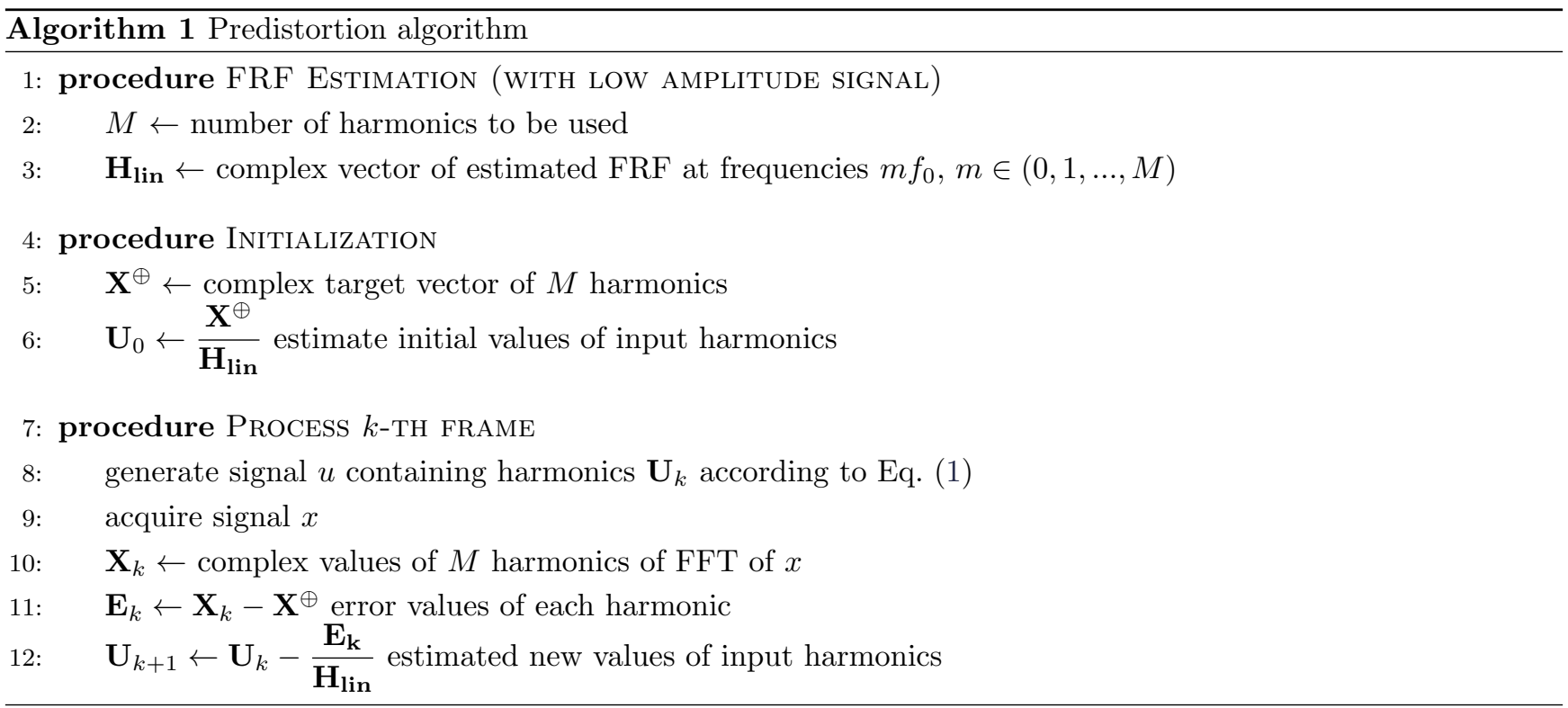

The algorithm (Algorithm 1) aims at estimating the harmonic components $\left(U_{m}, \phi_{m}\right)$ of the input signal $u(t)$ to minimize a minimum mean square error (MMSE) between $x(t)$ and $x^{\oplus}(t)$, or equivalently between each $\left(X_{m}, \psi_{m}\right)$ and $\left(X_{m}^{\oplus}, \psi_{m}^{\oplus}\right)$. It is based on an idea initially proposed in reference [27]. If the nonlinear device has a dominant linear path, which is the case for of almost all the excitation devices used in acoustics and vibrations, the predistortion algorithm can be described in the three following steps.

FRF Estimation The (linear) Frequency Response Function (FRF) $H_{l i n}(f)$ of the (nonlinear) excitation device can be estimated using the small-signal signal. Suppose, that the fundamental of the input signal $u(t)$ to 
be predistorted is $f_{0}$. Then, as the goal of the predistortion algorithm is to modify the harmonics (level $U_{m}$ and phase $\left.\phi_{m}\right)$ of $u(t)$, clearly the multiple integers of $f_{0}$ have to be stored as the FRF vector $\mathbf{H}_{\text {lin }}=$ $\left[H_{l i n}(0), H_{l i n}\left(f_{0}\right), H_{l i n}\left(2 f_{0}\right), \ldots, H_{l i n}\left(M f_{0}\right)\right]$.

For this first step, a random-phase multi-tone signal of low amplitude, consisting of $M$ harmonics $u_{F R F}(t)=$ $\sum_{m=0}^{M} \cos \left(m 2 \pi f_{0} t+\phi_{\text {rand }}\right), \phi_{\text {rand }}$ being chosen randomly, is used in the experimental part of this paper. Note that any signal allowing the FRF estimation, such as step-by-step sine-wave, maximum-length sequence [28], or a swept-sine signal [29] can also be considered.

Initialization A target vector $\mathbf{X}^{\oplus}=\left[X_{0}^{\oplus}, X_{1}^{\oplus} e^{j \psi_{1}^{\oplus}}, X_{2}^{\oplus} e^{j \psi_{2}^{\oplus}}, \ldots, X_{M}^{\oplus} e^{j \psi_{M}^{\oplus}}\right]$, consisting of a DC component and $M$ harmonics, defines the desired target signal $x^{\oplus}(t)$ according to Eq. (3). In the case of a pure sinusoidal target signal $x^{\oplus \sin }(t)$, only one value of this vector is non-zero. In general, any combination of harmonic components can be chosen as shown in Sec. 3. Next, a first estimation of the values $\left(U_{m}, \phi_{m}\right)$ defining the input signal $u(t)$ (Eq. (1)) is performed as

$$
\left.U_{m} e^{j \phi_{m}}\right|_{\mathbf{1}}=\frac{X_{m}^{\oplus} e^{j \psi_{m}^{\oplus}}}{H_{l i n}\left(m f_{0}\right)}
$$

Processing of k-th frame The predistortion algorithm is then recursively computed on successive non-overlapping frames $\mathbf{k}$ of $N$ samples. For each frame $\mathbf{k}$, we acquire $N$ samples of the signal $x(t)$ (sampled at frequency $f_{s}$ ) and we calculate its DFT. We impose $f_{0}$ to be one of spectral lines of the DFT and $M f_{0}$ to be lower than $f_{s} / 2$. We store the DC component $X_{0}$ and harmonic components $X_{m} e^{j \psi_{m}}$ of the DFT vector as $\mathbf{X}_{\mathbf{k}}=\left[X_{0}, X_{1} e^{j \psi_{1}}, X_{2} e^{j \psi_{2}}, \ldots, X_{M} e^{j \psi_{M}}\right]_{\mathbf{k}}$.

Next we note the complex-valued residual of harmonics $m \in[0, M]$

$$
\left.E_{m}\right|_{\mathbf{k}}=\left.X_{m} e^{j \psi_{m}}\right|_{\mathbf{k}}-X_{m}^{\oplus} e^{j \psi_{m}^{\oplus}}
$$

The algorithm is then based on subtracting $E_{m} / H\left(m f_{0}\right)$ from frame $\mathbf{k}$ to frame $\mathbf{k}+\mathbf{1}$ and consists then in writing

$$
\left.U_{m} e^{j \phi_{m}}\right|_{\mathbf{k}+\mathbf{1}}=\left.U_{m} e^{j \phi_{m}}\right|_{\mathbf{k}}-\frac{X_{m} e^{j \psi_{m}}-X_{m}^{\oplus} e^{j \psi_{m}^{\oplus}}}{H_{l i n}\left(m f_{0}\right)}
$$

Lastly, the signal $u(t)$ of the $\mathbf{k}+\mathbf{1}$ frame is calculated using Eq. (1) and next frame $(\mathbf{k}+\mathbf{1})$ is processed.

Note, that this method is simplification of the original technique [27] in which a second iterative loop to phase estimation is placed inside the main loop. Since, we know the output complex spectra (amplitude $X_{m}$ and phase $\psi_{m}$ ) the phase $\phi_{m}$ of injected harmonics can be estimated directly from Eqs. (5 and 7) without any other iterative loop.

\section{Experimental Validation}

For testing the predistortion algorithm presented in previous section we perform several experiments on an LDS V406 Brüel \& Kjær shaker driven by a D-Premier Devialet amplifier and loaded by a metallic rod of mass $23.5 \mathrm{~g}$ (Fig. 3). The first experiment demonstrates the ability to deliver a pure sinusoidal mechanical displacement (or acceleration) of the shaker with a high amplitude approaching its physical limits. The second experiment focuses on the generation of spectrally pure inter-modulation and multi-tone test signal. Finally, the last experiment presents the ability of the method to create any periodic form of the acceleration signal $x(t) \approx x^{\oplus}(t)$. In all these experiments, the reference signal $x(t)$ (Fig. 3) is measured using a PCB 352C22 accelerometer and PCB 428C sensor signal conditioner. The DAQ used for generating and acquiring signals is an RME Fireface 400 sound card with sampling frequency $f_{s}=48 \mathrm{kHz}$. The length of each frame during the real-time processing is $N=4800$ samples. $M=50$ components 


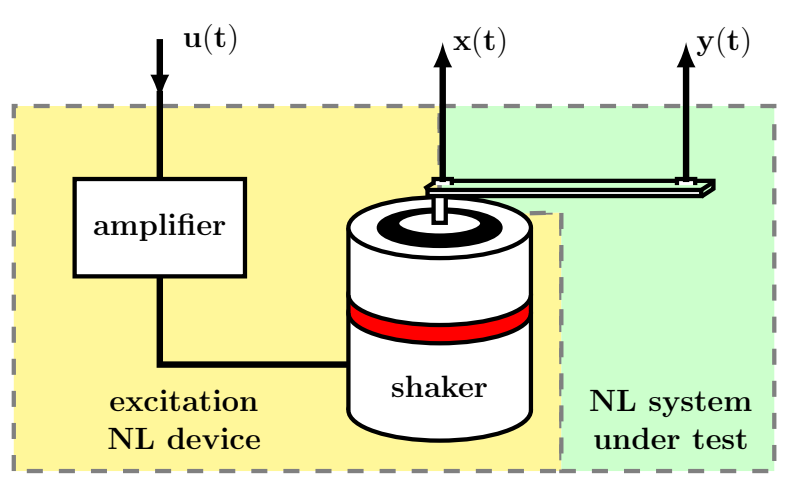

(a)

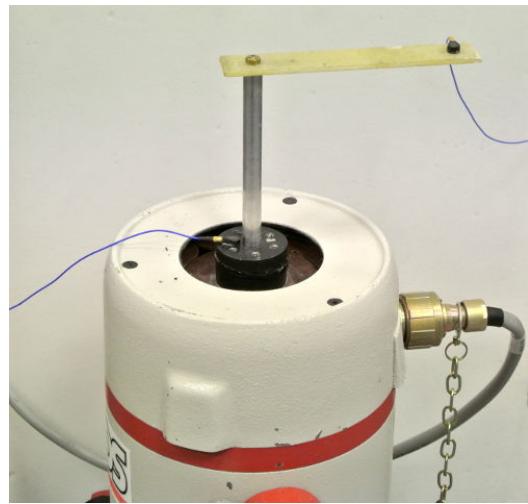

(b)

Figure 3. (a) Experimental setup using a shaker to generate vibration of the NL system under test and an accelerometer to measure the reference signal $x(t)$ to be corrected by means of predistortion of signal $u(t)$. Since the focus of the paper is distortion cancellation for the signal $x(t)$, the signal $y(t)$ is not used in the following. (b) Photo of the experimental setup.

are corrected at each iteration $\mathbf{k}$. The power spectra depicted in the figures below are calculated from signals with duration of 1 second.

Before each measurement, the FRF vector $\mathbf{H}_{\text {lin }}$ of the shaker is estimated using small level multi-tone signal with $M=50$ harmonics as explained in section 2.2. The level of the multi-tone signal is chosen to be at least $20 \mathrm{~dB}$ under the maximal limit of the shaker so that higher harmonics and the intermodulation products do not appear in the output signal (acceleration) of the shaker, but at least $20 \mathrm{~dB}$ over the noise level so that the estimated vector $\mathbf{H}_{\text {lin }}$ FRF provides a meaningful result.

\subsection{Pure sinusoidal mechanical displacement for vibration analysis}

In the first example, the shaker is excited by a sinusoidal signal $(50 \mathrm{~Hz}$ for the first part of the experiment and $200 \mathrm{~Hz}$ for the second part). For the experiment with $50 \mathrm{~Hz}$, the acceleration amplitude equals $500 \mathrm{~m} / \mathrm{s}^{2}$ (54 dB ref to $1 \mathrm{~m} / \mathrm{s}^{2}$ ), corresponding to both a $10 \mathrm{~mm}$ displacement amplitude and a $1.56 \mathrm{~m} / \mathrm{s}$ velocity amplitude. Since these values reach the physical limits of the shaker (the velocity limit of the shaker given by the manufacturer being $\pm 1.78 \mathrm{~m} / \mathrm{s}$ ), the vibration generated by the shaker and measured by the accelerometer as $x(t)$ is highly distorted, as shown in Fig. 4(a) (red solid line). The power spectra of the signal, depicted in red color in Fig. 4(b), shows an important amount of harmonic components created by the shaker. The fundamental to higher harmonic ratio is between $35 \mathrm{~dB}$ and $45 \mathrm{~dB}$ up to the $15^{\text {th }}$ harmonic.

The predistortion algorithm described in Sec. 2 needs four iterations to suppress all the higher harmonics from the signal $x(t)$. Regarding the higher-order harmonics cancellation process, the level of the first five harmonics is depicted in Fig. 4(c) as a function of time. Each iteration takes 0.3 second (one frame for update and two frames to ensure a steady-state response). The results show that, after four iterations (1.2 seconds), the fundamental to higher harmonic ratio is approximately $100 \mathrm{~dB}$ for all the higher harmonics. This is also verified by the power spectrum of the corrected acceleration signal $x(t) \approx x^{\oplus}(t)$ depicted in Fig. 4(d). All the higher harmonics are hidden in the background noise $100 \mathrm{~dB}$ below the fundamental harmonic. Note also, that the phase of the corrected signal (blue dashed line in Fig. 4(a)) is shifted. This is due to the fact that the target signal $x^{\oplus}(t)$ is defined as a cosine wave while the signal $x(t)$ (the one with no predistortion) is phase shifted by the system under test. In other words the algorithm corrects also the fundamental harmonic so that it matches perfectly the target, including the zero phase.

When applying the $200 \mathrm{~Hz}$ sinusoidal signal to produce a slightly lower acceleration (50 dB ref to $1 \mathrm{~m} / \mathrm{s}^{2}$, 
equivalent to an acceleration of $316 \mathrm{~m} / \mathrm{s}^{2}$ and a velocity of $0.25 \mathrm{~m} / \mathrm{s}$ ) the shaker operates much lower than its physical limits and the generated acceleration is considerably less distorted, as depicted in Fig. 5(a)) and Fig. 5(b). The fundamental to second and third harmonic ratios are $35 \mathrm{~dB}$ and $45 \mathrm{~dB}$ respectively. Once again, after four iterations, the predistortion algorithm success to suppress all the higher harmonic components (Fig. 5(c-d)).

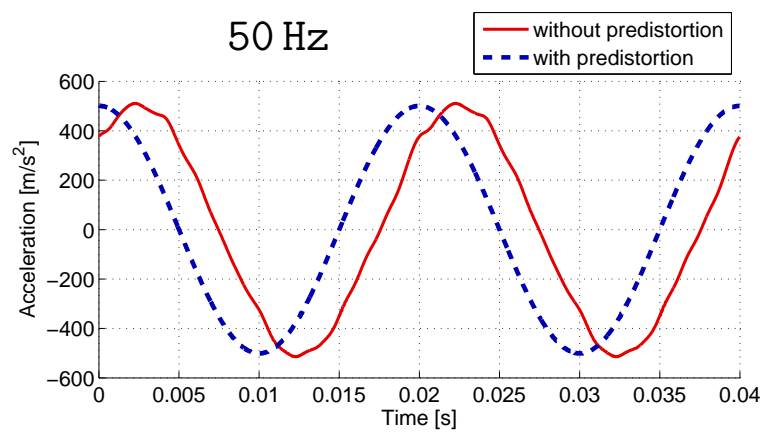

(a)

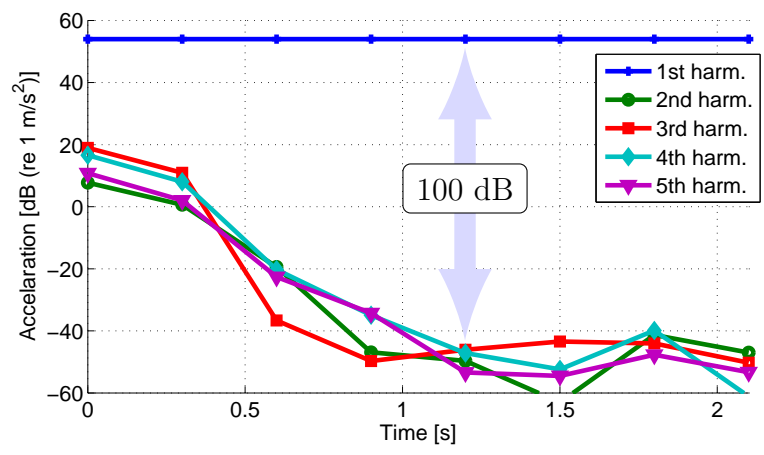

(c)

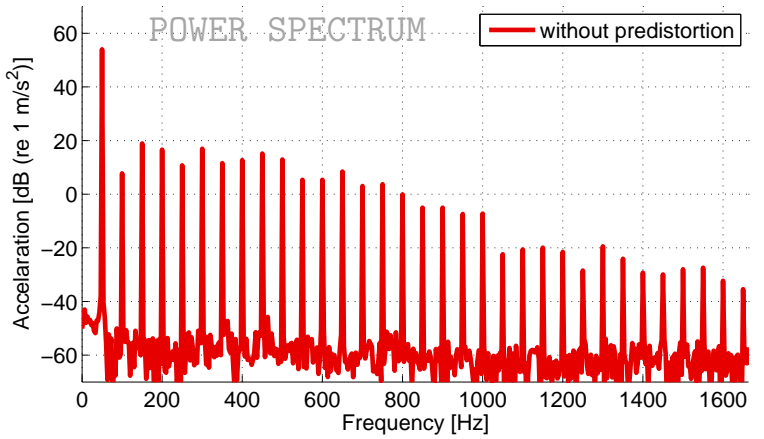

(b)

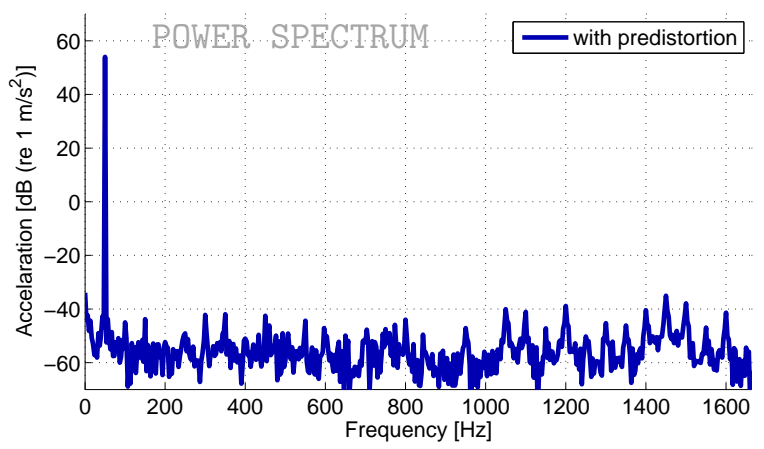

(d)

Figure 4. Case of pure sinusoidal $50 \mathrm{~Hz}$ experiment. Plot (a) shows the time domain acceleration waveforms $x(t)$ without (red solid line) and with (blue dashed line) predistortion algorithm. Plot (b) shows the power spectrum of the original (without predistortion) generated acceleration $x(t)$, whilst plot (d) shows the power spectrum of the generated acceleration $x(t)$ using the predistortion algorithm. The convergence of first five harmonics is depicted in plot (c).

\subsection{Pure two-tone and multi-tone generation}

The second experiment is focused on generating both a two-tone and a multi-tone acceleration signal. The twotone signal consists of two components $(450$ and $500 \mathrm{~Hz}$ ), each with a target amplitude of $45 \mathrm{~dB}$. The signal $x(t)$ generated by the shaker without the predistortion is shown in red in Fig. 6(a). As expected, many other harmonic components, as well as inter-modulation components, are contained in the spectra. After applying the predistortion algorithm, all undesirable harmonic components are removed from the acceleration which approximately equals the target signal $x(t) \approx x^{\oplus}(t)$ as depicted in blue line in Fig. 6(c)).

A similar result is achieved when generating a multi-tone acceleration consisting of seven components from 450 to $1650 \mathrm{~Hz}$. Spectral components other than the desired ones, created by the excitation device, are also observed in the spectra of the acquired acceleration signal $x(t)$ (Fig. 6(b)). After applying the predistortion algorithm, the undesirable spectral components are suppressed as depicted in Fig. 6(d). 


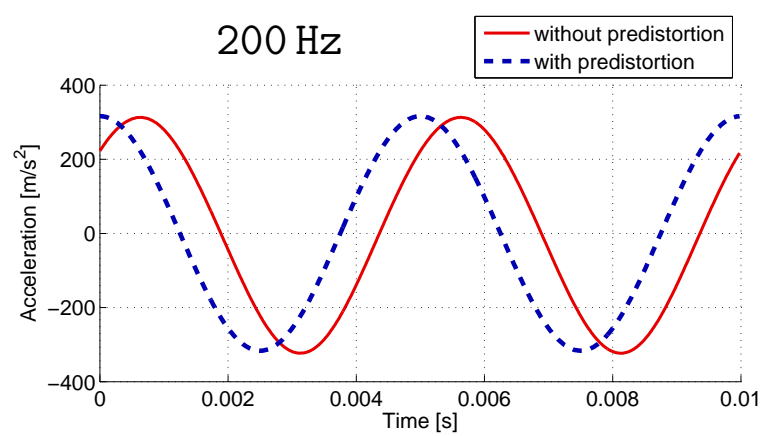

(a)

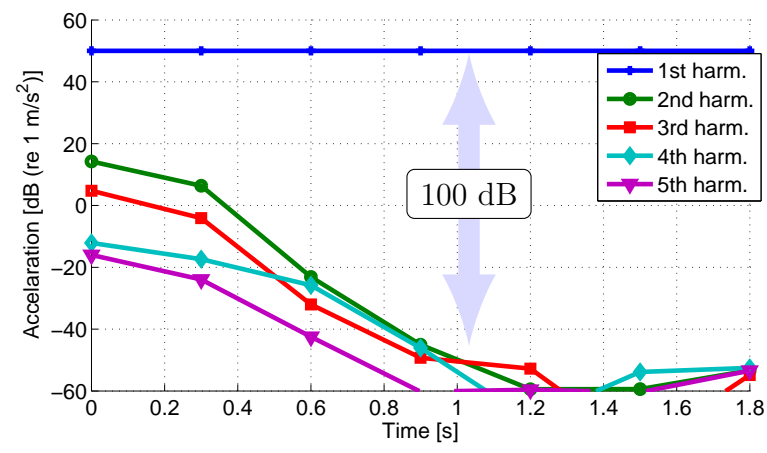

(c)

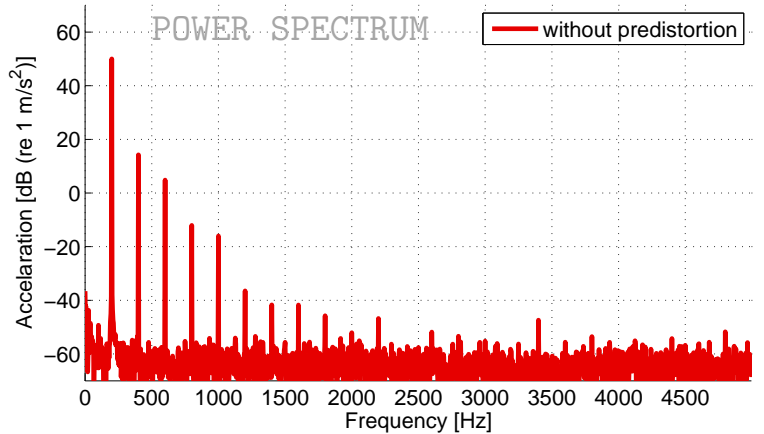

(b)

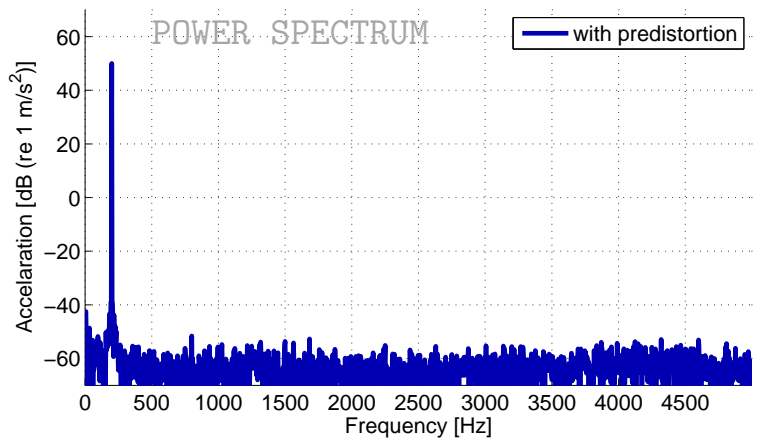

(d)

Figure 5. Case of pure sinusoidal $200 \mathrm{~Hz}$ experiment. Plot (a) shows the time domain acceleration waveforms $x(t)$ without (red solid line) and with (blue dashed line) predistortion algorithm. Plot (b) shows the power spectrum of the original (without predistortion) generated acceleration $x(t)$, whilst plot (d) shows the power spectrum of the generated acceleration $x(t)$ using the predistortion algorithm. The convergence of first five harmonics is depicted in plot (c).

\subsection{Generation of arbitrary signal shape}

The target signal $x^{\oplus}(t)$ for the last experiment is a triangle periodic acceleration $x^{\oplus}(t)=x^{\triangle}(t)$ with amplitude $A_{0}$ $=400 \mathrm{~m} / \mathrm{s}^{2}$ and fundamental frequency $f_{0}=50 \mathrm{~Hz}$. This particular example shows the ability of the method to generate any arbitrary periodic shape of the targeted acceleration signal $x^{\oplus}(t)$. This target signal may be expanded in a Fourier series as

$$
x^{\oplus}(t)=x^{\triangle}(t)=A_{0} \frac{8}{\pi^{2}} \sum_{m=1,3,5, \ldots}^{M} \frac{(-1)^{\frac{m-1}{2}}}{m^{2}} \sin \left(\frac{m 2 \pi f_{0} t}{L}\right),
$$

where $M=19$ components are chosen to approximate triangle periodic signal.

In Fig. 7, two measured acceleration signals are plotted. The red solid line signal corresponds to $x(t)$ for a triangle signal input $u(t)$, while the blue dashed line corresponds to $x(t) \approx x^{\oplus}(t)$ after applying the predistortion algorithm. 


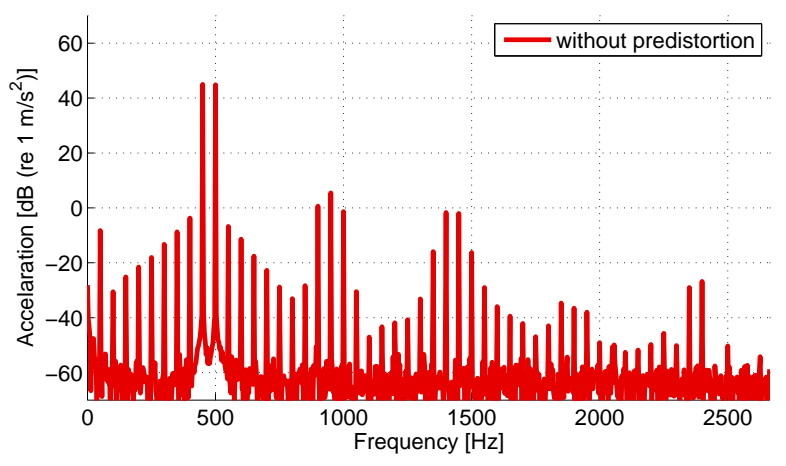

(a)

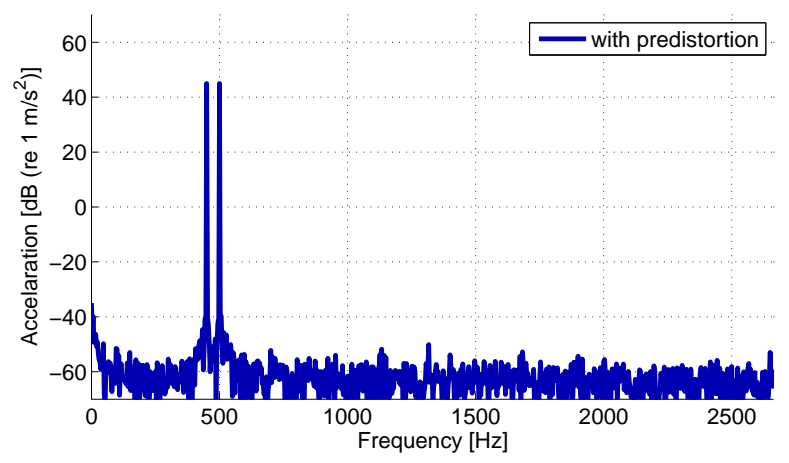

(c)

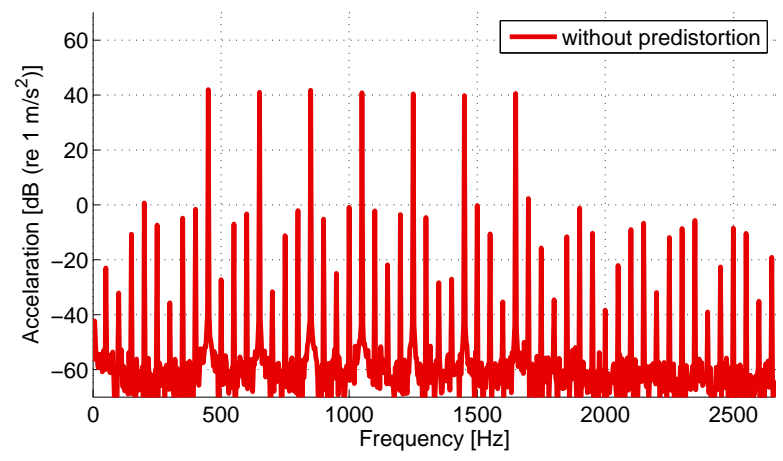

(b)

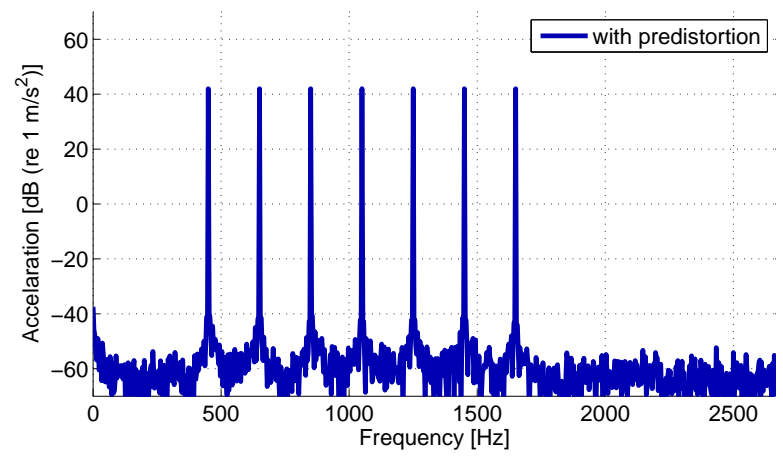

(d)

Figure 6. Power spectra for the two-tone and multi-tone experiments. (a) and (b): power spectra of the two-tone and multi-tone acceleration signals $x(t)$ without the predistortion. (c) and (d): power spectra of both signals after applying the predistortion algorithm.

\section{Discussion}

The experimental tests provided in previous section show that the predistortion technique can be applied in very effective manner (time and precision). It should be noted that the method presented in this paper does not require any special signal processing skills. The only adaptive procedure consists in adapting harmonic components (amplitude and phase) of the input signal in each processed frame. It is thus very fast and easy to implement.

\subsection{Limits of the proposed method}

The simplicity of the method is conditioned by the assumption that the excitation device has a dominant linear path. Consequently, a disadvantage of the method is its ineffectiveness when applied to non-linear excitation devices that do not obey this hypothesis. The assumption of the dominant linear path can be verified by applying a sinusoidal signal with levels low below the power limits of the device and measuring the total harmonic distortion (THD). If a dominant linear path is present in the system the THD remains very low $(\approx 1 \%)$. If the THD exceeds tens of percent even for small signals, a dominant linear path is probably not present in the system. However, a dominant linear path assumption applies to a large majority of excitation devices such as shakers, loudspeakers or compression drivers, which are used to excite many non-linear systems in acoustic and vibration experiments. Since the paper is addressed mainly to the experimentalists working with such devices, and since these devices are conceived by the manufacturers to behave as linearly as possible, the assumption of a dominant linear path should be satisfied for almost all these cases. 


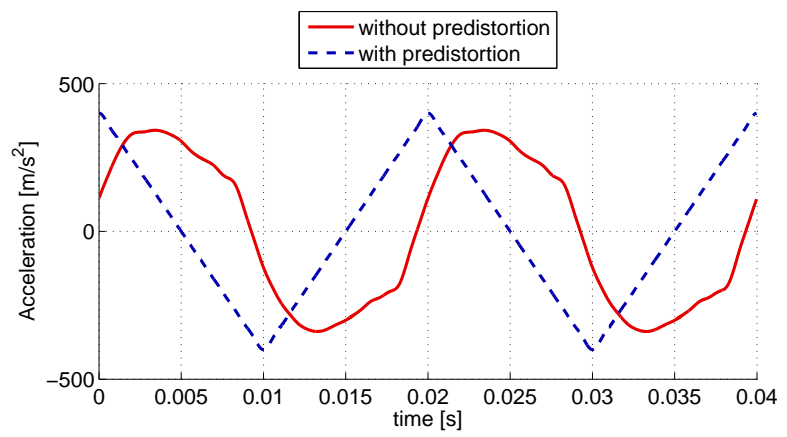

Figure 7. Waveforms of the triangular-like acceleration generated without (red solid line) and with (blue dashed line) predistortion algorithm.

Another limitation of the method is related to the physical limitations of the non-linear device. The desired amplitude of the target signal $x^{\oplus}(t)$ has obviously its own limits. A shaker, for example, cannot provide a displacement greater than a certain given value of maximum allowable displacement, nor can a small loudspeaker provide sound pressure levels beyond its physical limits. On the other hand, as shown in the experimental part of this paper, even if the target amplitude value is close to the physical limits, the method works fast and very accurately.

There are also limits related to the external disturbing signals. If, for any reason, the output signal contains a harmonic-like signal decorrelated from the input signal, the problems of stability may arise. A typical example of this problem is a presence of $50 \mathrm{~Hz}$ or $60 \mathrm{~Hz}$ derived from the power line in the measured signals. If the target signal contains a harmonic that matches this frequency (or its higher harmonics) and if the amplitude of this external disturbance is not negligible, the calculated correction can be under- or over-estimated.

Therefore, the robustness and stability of this method depend on two main assumptions: there is a dominant linear path in the system and there are no harmonic disturbances at the output that could coincide with the harmonics of the device's output signal. If one of these two is not satisfied, other methods, e.g. the ones based on adaptive filtering, should be consider.

Compared to other methods based on adaptive filtering [18], no adaptive filters, such as notch filters, that changes its weights adaptively are used in this method. The harmonic components are added adaptively to the input signal and thus, only the harmonic components that are defined in the target spectra can be rejected (or modified to match the target). Sub-harmonic, non-harmonic (super-harmonic), or intermodulation spectral components can be also easily cancelled out if their frequencies are included in the target spectra (see the example with intermodulation distortion in Fig. 6). Compared to the classical adaptive filters, this method can not adapt to any undesired frequency (which is not defined in the target spectra), on the other hand, it cancels out the undesired components defined in the target spectra quickly and effectively.

\subsection{Applications}

The technique proposed in this paper is therefore important for two types of studies. First of all, for studies in which a non-linear device under test is to be analysed or in which a decision based on the analysis of non-linear distortion is to be taken, e.g. non-destructive testing of (un)damaged materials [30]. If no predistortion is applied to the excitation device, the generated nonlinearities in the device under test (e.g. a damaged material) usually originate from both the exciter and the device under test. Consequently, an intact material can be classified as damaged. The proposed algorithm cancels out all the undesired harmonic components form the output signal of the excitation device (at the input of the device under test) and consequently also some of the harmonic components at the output (those originated from the excitation device). The remaining harmonic components at the output of the device under test are thus originated only from the device under test itself. Consequently, using this technique 
an intact material cannot be classified as damaged and the nonlinearities studied form the analysis of the output signal of the device under test are associated only with this device under test.

Second, for studies in which a nonlinear model of the device under test is identified. If no predistortion is applied and if the excitation signal is distorted due to the excitation device, complex nonlinear models and techniques that are used to describe the nonlinear behaviour of nonlinear systems under test using different approaches (parametric [31] or nonparametric [32]), based on different models such as Generalized Hammerstein model [33, 34], Volterra model [35, 36], or describing functions [37], can lead to erroneous results. The predistortion algorithm described in this article can be easily applied to these identification techniques for greater accuracy of estimated models.

\section{Conclusion}

In this paper, a simple et efficient method has been proposed, which consists in predistorting the excitation signal in order to remove higher undesirable harmonics generated by excitation device. The experimental results show that the shaker used with the predistortion algorithm is able to produce spectrally pure signals such as sinusoidal, two-tone, multi-tone, triangle, or any shape which could be represented by a finite sum of Fourier components, with a very large amplitude range approaching the limitations of the shaker. The predistortion algorithm is very simple and efficient, and it can be applied very easily using a scripting language such as Matlab, Octave, or Python, that can be used to control the data acquisition device connected to the excitation device and to the measurement setup.

The predistortion method is based on the assumption that a dominant linear path is present in the system to be corrected. Such an assumption is satisfied for almost all the excitation devices used in acoustics and vibrations such shakers, loudspeaker, or compression drivers. As shown in the experimental part of this paper, a spectral purity approaching $100 \mathrm{~dB}$ is achieved in few iterations $(\approx 1 \mathrm{~s})$ even if the excitation device works near its power limits.

This simple and highly efficient predistortion method thus addresses to the engineers and researchers working with experimental setups in which high amplitude signals need to be generated, usually for nonlinear system identification or nonlinear characterization of acoustical or mechanical systems under test. 


\section{References}

[1] N. Quaegebeur, A. Chaigne, Nonlinear vibrations of loudspeaker-like structures, Journal of Sound and Vibration 309 (1) (2008) 178-196.

[2] A. Saraswat, N. Tiwari, Modeling and study of nonlinear effects in electrodynamic shakers, Mechanical Systems and Signal Processing 85 (2017) 162-176.

[3] M. F. Hamilton, D. T. Blackstock, et al., Nonlinear acoustics, Vol. 427, Academic press San Diego, 1998.

[4] M. A. Rashidifar, Nonlinear Vibrations of Cantilever Beams and Plates, Anchor Academic Publishing, 2015.

[5] M. Bednarik, P. Konicek, Propagation of quasiplane nonlinear waves in tubes and the approximate solutions of the generalized burgers equation, The Journal of the Acoustical Society of America 112 (1) (2002) 91-98.

[6] D. S. Antao, B. Farouk, High amplitude nonlinear acoustic wave driven flow fields in cylindrical and conical resonators, The Journal of the Acoustical Society of America 134 (2) (2013) 917-932.

[7] M. Amabili, M. Pellegrini, M. Tommesani, Experiments on large-amplitude vibrations of a circular cylindrical panel, Journal of Sound and Vibration 260 (3) (2003) 537-547.

[8] P. Balasubramanian, G. Ferrari, M. Amabili, Z. J. G. N. del Prado, Experimental and theoretical study on large amplitude vibrations of clamped rubber plates, International Journal of Non-Linear Mechanics (2016) -doi:http://dx.doi.org/10.1016/j.ijnonlinmec.2016.12.006.

[9] J.-S. Pei, A. Smyth, E. Kosmatopoulos, Analysis and modification of volterra/wiener neural networks for the adaptive identification of non-linear hysteretic dynamic systems, Journal of Sound and Vibration 275 (3) (2004) 693-718.

[10] C.-Y. Chang, F.-B. Luoh, Enhancement of active noise control using neural-based filtered-x algorithm, Journal of Sound and Vibration 305 (1) (2007) 348-356.

[11] E. Abd-Elrady, L. Gan, Direct linearization of weakly nonlinear volterra systems using adaptive linear and nonlinear fir filters, IFAC Proceedings Volumes 42 (10) (2009) 646-651.

[12] K. Seki, M. Iwasaki, M. Kawafuku, H. Hirai, K. Yasuda, Adaptive compensation for reaction force with frequency variation in shaking table systems, IEEE Transactions on Industrial Electronics 56 (10) (2009) $3864-3871$.

[13] J. Yao, H. Yan, R. Xiao, D. Di, G. Jiang, S. Gao, H. Yu, Sinusoidal acceleration harmonic estimation using the extended kalman filter for an electro-hydraulic servo shaking table, Journal of Vibration and Control 21 (8) (2015) 1566-1579. doi:10.1177/1077546313499392.

[14] Y. Jianjun, H. Shenghai, F. Wei, H. Junwei, Harmonic cancellation for electro-hydraulic servo shaking table based on lms adaptive algorithm, Journal of Vibration and Control 17 (12) (2011) 1862-1868.

[15] L. P. De Oliveira, B. Stallaert, K. Janssens, H. Van der Auweraer, P. Sas, W. Desmet, Nex-lms: A novel adaptive control scheme for harmonic sound quality control, Mechanical Systems and Signal Processing 24 (6) (2010) 1727-1738.

[16] M. Buchard, B. Paillard, An alternative feedback structure for the adaptive active control of periodic and time-varying periodic disturbances, Journal of Sound and Vibration 210 (4) (1998) 517-527. 
[17] M. Bodson, Rejection of periodic disturbances of unknown and time-varying frequency, International Journal of Adaptive Control and Signal Processing 19 (2-3) (2005) 67-88.

[18] J. Yao, M. Dietz, R. Xiao, H. Yu, T. Wang, D. Yue, An overview of control schemes for hydraulic shaking tables, Journal of Vibration and Control 22 (12) (2016) 2807-2823.

[19] P. Mattavelli, F. P. Marafao, Repetitive-based control for selective harmonic compensation in active power filters, IEEE Transactions on Industrial Electronics 51 (5) (2004) 1018-1024.

[20] Y. Pasco, O. Robin, P. Bélanger, A. Berry, S. Rajan, Multi-input multi-output feedforward control of multiharmonic gearbox vibrations using parallel adaptive notch filters in the principal component space, Journal of Sound and Vibration 330 (22) (2011) 5230-5244.

[21] L. Ucun, J. Salášek, Hosidf-based feedforward friction compensation in low-velocity motion control systems, Mechatronics 24 (2) (2014) 118-127.

[22] H. Malloug, M. J. Barragan, S. Mir, Evaluation of harmonic cancellation techniques for sinusoidal signal generation in mixed-signal bist, in: Mixed-Signal Testing Workshop (IMSTW), 2015 20th International, IEEE, 2015, pp. 1-6.

[23] R. Enokida, I. Takewaki, D. Stoten, A nonlinear signal-based control method and its applications to input identification for nonlinear simo problems, Journal of Sound and Vibration 333 (24) (2014) 6607-6622.

[24] A. Singh, J. E. Scharer, J. H. Booske, J. G. Wohlbier, Second-and third-order signal predistortion for nonlinear distortion suppression in a twt, IEEE Transactions on Electron Devices 52 (5) (2005) 709-717.

[25] A. M. Eltamaly, A novel harmonic reduction technique for controlled converter by third harmonic current injection, Electric Power Systems Research 91 (2012) 104-112.

[26] A. Novak, B. Maillou, P. Lotton, L. Simon, Nonparametric identification of nonlinear systems in series, IEEE Transactions on Instrumentation and Measurement 63 (8) (2014) 2044-2051.

[27] D. Rabijns, W. Van Moer, G. Vandersteen, Spectrally pure excitation signals: only a dream?, IEEE Transactions on Instrumentation and Measurement 53 (5) (2004) 1433-1440.

[28] J. Vanderkooy, Aspects of mls measuring systems, Journal of the Audio Engineering Society 42 (4) (1994) 219-231.

[29] A. Novak, P. Lotton, L. Simon, Synchronized swept-sine: Theory, application, and implementation, Journal of the Audio Engineering Society 63 (10) (2015) 786-798.

[30] A. Novak, M. Bentahar, V. Tournat, R. El Guerjouma, L. Simon, Nonlinear acoustic characterization of microdamaged materials through higher harmonic resonance analysis, NDT\&E International 45 (1) (2012) 1-8.

[31] Ö. Arslan, M. Aykan, H. N. Özgüven, Parametric identification of structural nonlinearities from measured frequency response data, Mechanical Systems and Signal Processing 25 (4) (2011) 1112-1125.

[32] G. Kenderi, A. Fidlin, Nonparametric identification of nonlinear dynamic systems using a synchronisationbased method, Journal of Sound and Vibration 333 (24) (2014) 6405-6423.

[33] A. Novak, L. Simon, F. Kadlec, P. Lotton, Nonlinear system identification using exponential swept-sine signal, IEEE Transactions on Instrumentation and Measurement 59 (8) (2010) 2220-2229. 
[34] M. Rébillat, R. Hennequin, E. Corteel, B. F. Katz, Identification of cascade of hammerstein models for the description of nonlinearities in vibrating devices, Journal of Sound and Vibration 330 (5) (2011) 1018-1038.

[35] A. Chatterjee, N. S. Vyas, Non-linear parameter estimation with volterra series using the method of recursive iteration through harmonic probing, Journal of Sound and Vibration 268 (4) (2003) 657-678.

[36] T. Hélie, Volterra series and state transformation for real-time simulations of audio circuits including saturations: Application to the moog ladder filter, IEEE Transactions on Audio, Speech, and Language Processing 18 (4) (2010) 747-759.

[37] P. Nuij, O. Bosgra, M. Steinbuch, Higher-order sinusoidal input describing functions for the analysis of nonlinear systems with harmonic responses, Mechanical Systems and Signal Processing 20 (8) (2006) 1883-1904. 